\title{
Recent advances in the surgical management of hepatocellular carcinoma
}

\author{
Georgios K. Glantzounisa, Anastasia Karampaa, Dimitra V. Peristeria, George Pappas-Gogosa, \\ Kostas Tepelenis ${ }^{a}$, Petros Tzimas ${ }^{b}$, Dimitrios J. Cyrochristos ${ }^{a}$
}

University Hospital of Ioannina and School of Medicine, University of Ioannina, Ioannina, Greece

Abstract

\begin{abstract}
The incidence of hepatocellular carcinoma (HCC) is increasing, despite effective antiviral treatment for hepatitis B (HBV) and C virus infection and the application of preventive measures such as vaccination at birth against HBV infection. This is mainly due to the increase in metabolic syndrome and its hepatic components, nonalcoholic fatty liver disease and steatohepatitis. Liver resection and transplantation are the main treatment options, offering long-term survival and potential cure. In this review, the recent advances in the surgical management of HCC are presented. More specifically, the role of liver resection in the intermediate and advanced stages, according to the Barcelona Clinic Liver Cancer classification, is analyzed. In addition, the roles of minimally invasive surgery and of living-related liver transplantation in the management of patients with HCC are discussed. Finally, recent data on the role of molecular markers in the early diagnosis and recurrence of HCC are presented. The management of HCC is complex, as there are several options for each stage of the disease. In order for, each patient to get the maximum benefit, an individualized approach is suggested, in specialized liver units, where cases are discussed in multidisciplinary tumor boards.
\end{abstract}

Keywords Hepatocellular carcinoma, surgical management, intermediate and advanced stage, minimally invasive surgery, living-related liver transplantation

Ann Gastroenterol 2021; 34 (x): 1-13

\section{Introduction}

The incidence of hepatocellular carcinoma (HCC) is increasing worldwide. HCC is the fifth most common cancer and the second most common cause of death in men [1]. The highest incidence is in Eastern Asia. HCC usually develops in chronic liver disease where there is hepatic fibrosis, steatosis or cirrhosis. Hepatocarcinogenesis is a long process that involves several molecular mechanisms [2].

The main risk factors for developing $\mathrm{HCC}$ are chronic active infection with hepatitis $\mathrm{B}(\mathrm{HBV})$ and $\mathrm{C}(\mathrm{HCV})$ virus,

${ }^{\text {a} H P B ~ U n i t, ~ D e p a r t m e n t ~ o f ~ S u r g e r y ~(G e o r g i o s ~ K . ~ G l a n t z o u n i s, ~ A n a s t a s i a ~}$ Karampa, Dimitra V. Peristeri, George Pappas-Gogos, Kostas Tepelenis,

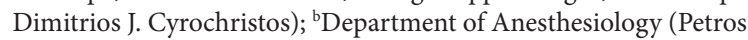

Tzimas), University Hospital of Ioannina and School of Medicine, University of Ioannina, Ioannina, Greece

\section{Conflict of Interest: None}

Correspondence to: Georgios K. Glantzounis, MD, PhD, FEBS, Professor of Surgery and Transplantation, Head of HPB Unit, Department of Surgery, School of Medicine, University of Ioannina, 45 110, Ioannina, Greece, e-mail: gglantzounis@uoi.gr

Received 30 October 2020; accepted 19 February 2021; published online 27 May 2021

DOI: https://doi.org/10.20524/aog.2021.0632 high alcohol intake, aflatoxin exposure, nonalcoholic fatty liver disease (NAFLD), and nonalcoholic steatohepatitis. Despite effective antiviral treatment for HCV and HBV, and preventive measures such as vaccination at birth against HBV infection, the incidence of HCC is increasing. The main reason for this is the increase in obesity, metabolic syndrome, and NAFLD [3].

Surgery (liver resection and transplantation) is the main treatment; it offers long-term survival with good quality of life and potential cure. In order to achieve better results with low perioperative morbidity and mortality, highly efficacious anesthesia is required. There are many challenges and key issues in the management of anesthesia in patients who undergo hepatectomy for HCC [4]. It is critical to identify preoperative risk factors, such as portal hypertension and cirrhosis, because the underlying liver disease can lead to significant complications [5]. Several effects on circulation, lung function, renal function and coagulation have to be expected [5]. As a rule, the restriction of fluids and low central venous pressure are used to limit blood loss and transfusion requirements [5]. Pain management strategies have long been debated, with the primary focus being on epidural analgesia [6]. Although thoracic epidural anesthesia and analgesia are commonly used for hepatectomies, there are concerns over their safety in view of postoperative coagulation derangements [6], especially in patients with HCC and often underlying liver cirrhosis. 
Alternative methods of postoperative analgesia, such as intrathecal morphine have also been suggested [6]. Intrathecal morphine appears to have several advantages, particularly in the context of enhanced recovery after surgery (ERAS) programs [7]. Indeed, ERAS programs have been associated with lower morbidity and a shorter postoperative stay [7].

The present review will focus on the recent advances in the surgical management of HCC. The main areas that will be covered are: a) the role of surgery in the intermediate and advanced stages and the importance of the individualized approach through multidisciplinary meetings; b) the expanding role of minimally invasive surgery (MILR) (laparoscopic and robotic liver resections); c) the role of living donor liver transplantation (LDLT) in the management of HCC; and d) the significance of molecular markers in the early diagnosis of HCC and of recurrence.

\section{Surgical management of HCC in intermediate and advanced stage}

The staging of HCC is complex: it includes assessment of liver function, tumor extent, presence of metastases and the patient's general condition, as the surgical management requires major operations (hepatectomy or liver transplantation [LT]). The Barcelona Clinic Liver Cancer (BCLC) staging system is the most commonly accepted system for prognosis and study comparisons. However, it is questioned in the western world by the hepatobiliary surgical community, regarding treatment allocation, and is not used in Asia [8]. The main questions arise in the intermediate and advanced stages with vascular involvement, where the BCLC system proposes only conservative treatments.

Transarterial chemoembolization (TACE) with lipiodol is the standard of care for patients with intermediated stage HCC (>2 lesions, $>3 \mathrm{~cm}$ each) according to the BCLC criteria $[9,10]$. Two randomized controlled trials have shown a median survival of 18-27 months [11,12]. The use of drug-eluting microspheres has facilitated the more controlled release of chemotherapeutic agents and is associated with fewer systemic side effects. In a recent single-center prospective phase II clinical trial the patients with intermediate stage HCC (BCLC B) had an overall survival of 26 months, while the 3- and 5-year survival rates were $36 \%$ and $2.7 \%$ respectively [13].

The proposal of TACE, as the only management option for the intermediate stage, in the BCLC algorithm has been questioned heavily by the international hepatobiliary surgical community. An observational multicenter study that included patients from Europe, USA and Asia showed that $36 \%$ of patients who undergo liver resection for HCC were classified as BCLC $B$ and a 5-year overall survival rate of $57 \%$ was achieved [14]. However, at that point single HCCs $>5 \mathrm{~cm}$ were classified as BCLC B. To overcome this problem, our group conducted a systematic review, looking at the role of liver resection in the management of multinodular intermediate stage HCCs $(>2$, $>3 \mathrm{~cm}$ each). Twenty-three studies were selected that included 2412 patients with multinodular HCCs (Table 1). The majority of the studies were from east Asia. The median postoperative morbidity was $25 \%$ and the 90 -day mortality was $2.7 \%$. The median follow up was 27.6 months, median survival was 37 months, and 5-year survival 35\% [15]. There were significant differences in outcomes related with the number of lesions. Hepatectomy for up to 3 HCCs provided median survival of 47 months and 5-year overall survival of $49 \%$. On the other hand, for $>3$ HCCs the median survival was 18 months and the 5-year overall survival 23\%. Recently, the Pan-Asianadapted European Society for Medical Oncology (ESMO) clinical practice guidelines for the management of patients with intermediate and advanced/relapsed HCC have included liver resection as a reliable option for multinodular HCC [8].

Advanced stage (C), according to the BCLC staging system, is a very heterogenous group of patients, including HCCs with portal vein (PV) invasion and or extrahepatic spread (metastases). The liver function is preserved (ChildPugh B) and the performance status is good [10]. For this group of patients, systemic targeted therapy is the standard of care, according to BCLC criteria, with a life expectancy of $\geq 10$ months. Sorafenib, a multikinase inhibitor that targets vascular endothelial growth factor receptor, platelet-derived growth factor receptors, and RAF kinases signaling, is the standard of care $[8,10]$. Regorafenib is the standard of care, as second-line treatment, for patients with advanced HCC who have tolerated sorafenib but progressed. It is recommended in patients with well-preserved liver function and Eastern Cooperative Oncology Group performance status 0-1 [16]. Regorafenib improved overall survival versus placebo (10.6 vs. 7.8 months) [16]. The results in the advanced stage are not satisfactory and new treatments are required. For this reason, intensive research is being undertaken; new agents such as cabozantinib, ramucirumab and immunotherapy with nivolumab are being tested with varying results [8].

Table 1 Main data from a systematic review on the role of liver resection in the management of intermediate and advanced stage HCC [15]

\begin{tabular}{|c|c|c|c|c|c|c|c|}
\hline $\begin{array}{l}\text { HCC } \\
\text { STAGE } \\
\text { (BCLC } \\
\text { criteria) }\end{array}$ & $\begin{array}{l}\text { Patients } \\
\text { (n) }\end{array}$ & $\begin{array}{c}\text { Postoperative } \\
\text { morbidity }\end{array}$ & $\begin{array}{c}\text { Postoperative } \\
\text { mortality }\end{array}$ & $\begin{array}{l}\text { Median } \\
\text { survival } \\
\text { (months) }\end{array}$ & $\begin{array}{l}\text { 5-year } \\
\text { survival }\end{array}$ & $\begin{array}{l}\text { 5-year survival } \\
\text { (multinodular } \\
\leq 3 \text {, distal PV } \\
\text { thrombosis }{ }^{\star} \text { ) }\end{array}$ & $\begin{array}{l}\text { 5-year survival } \\
\text { (multinodular }>3, \\
\text { first-order branches } \\
\text { PV thrombosis }{ }^{* *} \text { ) }\end{array}$ \\
\hline Multinodular & 2412 & $25 \%$ & $2.7 \%$ (90 days) & 37 & $35 \%$ & $49 \%$ & $23 \%$ \\
\hline PVTT & 3659 & $33 \%$ & $2.7 \%$ (in-hospital) & 15 & $20 \%$ & $45 \%^{*}$ & $19 \%^{* *}$ \\
\hline
\end{tabular}


The main question is whether surgical treatment has a role in the advanced stage. It seems that it could have a role in patients with limited macrovascular invasion of the PV. The advanced stage is a very heterogeneous stage, including patients with limited macrovascular invasion, extended vascular invasion and extrahepatic metastases. An observational multicenter study with patients from Europe, USA and Asia showed that $14 \%$ of patients who undergo liver resection for HCC were classified as BCLC C, and a 5-year overall survival of 38\% was achieved [14]. There are different types of PV tumor thrombosis (PVTT), which also affect the extent of PVTT. There are 2 main classification systems, one of them proposed by the liver cancer study group of Japan [17]. According to this, tumor thrombus in distal and second order PV branches is classified as $\mathrm{Vp} 1$ and $\mathrm{Vp} 2$, respectively. The presence of tumor thrombus in the main right or left PV is classified as Vp3. In the main PV trunk, contralateral or both it is classified as Vp4 (Fig. 1). The second classification comes from China [18]. According to this classification, type 1 refers to tumor thrombus in segmental branches of the PV, and type II refers to the presence of thrombus in the right or left PV. In type III, tumor thrombus involves the main PV trunk and in type IV thrombus extends to the superior mesenteric vein.

In our systematic review mentioned above, we also looked at the role of liver resection in the management of advanced stage HCC [15]. Twenty-nine studies were selected that included 3659 patients who had HCC with macrovascular invasion (Table 1). The median postoperative morbidity was $33 \%$ and the in-hospital mortality $2.7 \%$. The median follow up was 25 months and the median survival 15 months. The 3 - and 5 -year survival rates were $33 \%$ and $20 \%$, respectively. Twelve studies reported separate survival data according to the stage of PVTT. The median 5-year survival for Vp1-2 patients was $45 \%$. The median 5-year survival was $19 \%$ for Vp3 PVTT and $14.5 \%$ for Vp4 PVTT patients. Only 4 studies originated from the West.

It seems that the favorable results reported by both eastern and western hepato-pancreato-biliary centers support the role of liver resection in patients with PVTT of the first (Vp1) and secondary (Vp2) branches. A revision of western countries' guidelines is required to fully recognize hepatic resection as a valid alternative for selected cases of advanced HCC [19]. Recently, the Pan-Asian adapted ESMO clinical practice guidelines for the management of patients with intermediate and advanced/relapsed HCC have included liver resection as an option for advanced stage HCC with intrahepatic macrovascular invasion without extrahepatic metastases [8]. Furthermore, a recent study from Japan showed that hepatic resection in patients with HCC and portal hypertension offers overall survival of $50 \%$, and the perioperative prophylactic management of portal hypertension increases the safety of the procedure, with low postoperative morbidity and mortality [20]. Also, as the treatment of HCC is complex and there are different management options for each stage, recent evidence suggests that discussion of HCC cases in a multidisciplinary tumor board should be mandatory, in order to reach the best therapeutic decision $[21,22]$.

\section{MILR in HCC (laparoscopic and robotic surgery)}

MILR is increasing globally; its wide acceptance has occurred in parallel with the increasing trend to perform limited resections for malignant lesions. Reich performed the first laparoscopic liver resection (LLR) for benign lesion in the early 1990s [23]. In highly specialized hepatobiliary centers, the percentage of MILR performed every year has increased by up to $50 \%$ over the last 2 decades, mainly for malignant lesions. In 2008 the Louisville Statement [24] introduced the indication of MILR for solitary lesions of $5 \mathrm{~cm}$ or less, located in liver segments II, III IVb, V, VI and requiring segmental resections or left lateral hepatectomy. The panel of experts suggested that major liver resections should be reserved for experienced surgeons in liver surgery and advanced laparoscopic surgery in specialized centers. The second international consensus conference held in Marioka in 2014 stressed the need for a formal educational structure for those interested in performing major LLR, because of the steep learning curve [25]. The third international meeting was held in Seoul, Korea, in 2016, with special focus on the role of laparoscopic liver donor hepatectomy. It was concluded that laparoscopic donor hepatectomy is increasing in both pediatric and adult LDLT. It was emphasized that for major donor hepatectomy more evidence is needed [26].

LLR is one of the most significant achievements in the field of liver surgery. For patients with resectable HCC in particular, LLR has several advantages over the open approach, as HCC usually develops on a liver with underlying disease. The main advantages are the lower incidence of postoperative liver failure and ascites, as the abdominal trauma is much smaller and the surgical stress significantly less [27]. A recent systematic review and meta-analysis compared the short- and long-term outcomes of laparoscopic and open liver resections for HCC. It concluded that LLR for HCC is feasible and offers better shortterm outcomes in respect of complication rate, blood loss and duration of hospital stay, and comparable long-term outcomes to the open approach [28]. Similar conclusions have been reached in another recent systematic review [29] and meta-analysis [30].

For suitable HCC patients selected for LLR, 3 important factors must be taken into account: the presence of cirrhosis, the location of the mass, and the size of the mass. Several studies have shown the feasibility of LLR for HCC in cirrhotic patients, which could also reduce complications and shorten hospital stay $[31,32]$. All these data support the message that LLR may be a viable alternative to an open procedure in patients with liver cirrhosis. As regards the location of the tumor, Zheng et al [33] presented 281 patients who underwent LLR for lesions located in posterior liver segments (I, IVa, VII, and VIII). Blood loss, complication rate, hospital stay and tumor recurrence were not significantly different in comparison to LLRs in anterolateral segments (II, III, IVb, V and VI), although the operation time was longer and the conversion rate higher. In experienced centers, the tumor location may not be a contraindication for the laparoscopic approach. It is well known that tumor size is a risk factor in both laparoscopic and open liver tumor resection. Retrospective studies that compared patients who underwent LLR for HCCs of $\geq 5$ and $<5 \mathrm{~cm}$ [34] indicated that the operation 


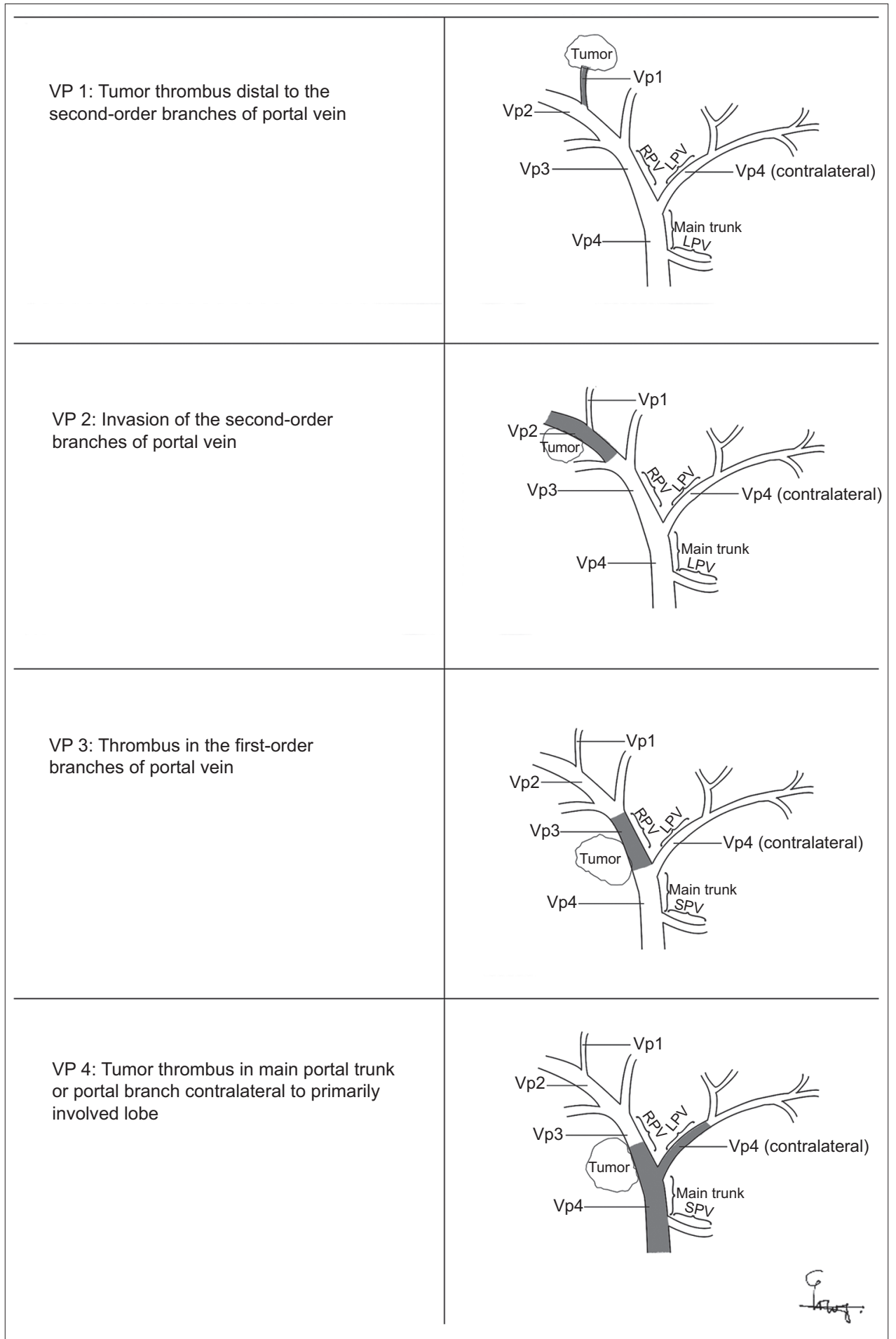

Figure 1 Portal vein tumor thrombosis classification according to the liver study group of Japan

HCC, hepatocellular carcinoma; HBV, hepatitis B; HCV, hepatitis C; NAFLD, nonalcoholic fatty liver disease; MILR: minimally invasive liver surgery; LDLT, living donor liver transplantation; LT, liver transplantation; BCLC, Barcelona Clinic Liver Cancer; TACE, Transarterial chemoembolization; $P V$, portal vein; LLR, laparoscopic liver resection; MC, Milan criteria; UCSF criteria, the University of California, San Francisco criteria; MELD, model for end-stage liver disease; DDLT, deceased donor liver transplantation; SFSS, small-for-size syndrome; AFP, Alpha-fetoprotein; AASLD, American Association for the Study of Liver Diseases; EASL, European Association for the Study of the Liver

time, conversion rate, blood loss, postoperative hospital stay, the disease-free survival, and the length of overall survival were comparable between the 2 groups. A recent study showed that laparoscopic left lateral segmentectomy for large HCCs $(>5 \mathrm{~cm})$ 
had better perioperative outcomes and equivalent oncologic outcomes compared to open resection [35]. A recent report from the international survey on the technical aspects of 4478 difficult LLRs (major hepatectomy, postero-superior segmentectomy, sectionectomy, living donor hepatectomy, tumor size $>10$ $\mathrm{cm}$, Child-Pugh B, combined with biliary reconstruction) concluded that most procedures are safe and feasible when conducted in specialized centers [36]. As mentioned above, LLR is advantageous in reducing blood loss and transfusion rate. An appropriate balance between $\mathrm{CO}_{2}$ pressure pneumoperitoneum and central venous pressure is essential to decrease bleeding and maintain stable hemodynamics.

Another application of MILR is laparoscopic ablation therapies for HCC lesions not eligible for a percutaneous approach (e.g., location in posterior segments) or surgical resection (affected general condition or liver function). Recent evidence suggests that laparoscopic ablation of liver lesions is safe and effective [37]. Furthermore, as tumor recurrence is very common after liver resection for HCC in chronic liver disease, the application of laparoscopic repeat liver resection after open or laparoscopic liver hepatectomy is safe and can be performed with favorable short-term outcomes in highly selected cases [38,39]. However, LLR remains a technically demanding procedure that requires surgical technology (CUSA, Ligasure device, harmonic scalpel, 3D high-definition camera) and an experienced surgical team. It seems that the use of new techniques, such as indocyanine green fluorescence imaging, will facilitate the safe performance of anatomic liver resections laparoscopically [40]. In conclusion, thanks to the efforts of both liver surgeons and medical engineers over the last 2 decades, LLR has become a technically feasible and reliable treatment for liver disease. A learning curve of 60 cases is required in order for surgeons to be able to perform high-quality LLR procedures.

On the other hand, the introduction of robotic surgery might fill the gaps of conventional laparoscopy. It was initially reported for liver operations in 2010 [41]. Robotic surgical systems allow easier access to deep intraparenchymal and postero-superior liver areas. At the same time, robotic surgery is characterized by flexibility, enabling curved parenchymal transection, which can only be reached with difficulty with the conventional straight laparoscopic instrument and camera system [42]. Therefore, lesions in the postero-superior segments of the liver, as well as those with major vascular involvement, could be more easily approached in robotic surgery. Robotic surgery facilitates a parenchymal-sparing liver resection, while at the same time it ensures less surgeon fatigue, especially in long procedures [43]. Although several studies have been published regarding robotic liver resection, showing its safety and feasibility, the comparison with open and conventional laparoscopic techniques is still in its infancy. It seems that robotic liver resection maintains the typical benefits of minimally invasive surgery, including shorter operative times, lower blood loss, and shorter hospital stays compared to open surgery, but its superiority over laparoscopy has not yet been proved [44-46]. The robotic technique has a shorter learning curve compared to laparoscopic hepatectomy [47]. On the other hand, robotic surgery has a much higher cost in comparison with LLR, without consistently evident superiority in terms of clinical benefit $[47,48]$. Furthermore, at present, robotic sources of energy (ultrasound, bipolar electrocautery) are inferior compared to those used in laparoscopy. In conclusion, the most significant clinical benefit of the robotic system over conventional laparoscopy is presumably the performance of minor resections in liver lesions in difficult locations (postero-superior segments). Also, the endo-wristed instruments make the robotic system appropriate for parenchymal-sparing resection, and during hilar dissection they allow parenchymal preservation, even for tumors close to a hepatic vein and portal pedicles [47]. Table 2 presents the main data from large studies from East and West relating to LLRs in patients with HCC [49-55].

\section{LDLT}

HCC is the most common tumor to be treated with whole organ transplantation, and it is listed as the primary indication in approximately $20 \%$ of LT worldwide [56,57]. The results of LT for HCC have improved drastically in the last 20 years, mainly because of better patient selection, and currently the recurrence rate ranges from $11-18 \%$ and the 5-year survival rate is more than $70 \%$ [58]. Since the introduction of the Milan criteria (MC) by Mazzaferro et al in 1996 (single tumor $<5 \mathrm{~cm}$ or up to 3 tumors, none of them larger than $3 \mathrm{~cm}$, no macrovascular invasion) the number of patients transplanted for HCC has seen significant growth, with long-term results comparable to those of non-HCC patients $[59,60]$. Moreover, many centers are making efforts to expand these selection criteria and have developed modified or extended guidelines in order to improve patient evaluation-the University of California, San Francisco (UCSF) criteria; Up-to-seven, Toronto; Hangzhou or Chengdu criteria in mainland China, etc.-in an effort to achieve satisfactory long-term survival after LT among those patients [61].

The indication for LT for HCC in terms of liver functional reserve, especially in western countries, is based on the model for end-stage liver disease (MELD) score, with additional points for those patients. Until now, LT has been offered to patients with $\mathrm{HCC}$ within the MC and preserved liver function [10]. However, there is a lack of potential donors for deceased donor LT (DDLT). A long waiting period is problematic for HCC patients [62]. The latest data indicate that many patients with HCC have a very low probability of receiving DDLT before tumor progression, because most deceased donor livers are allocated to patients with a high MELD score $(>30)$. The current waitlist dropout rate in Europe ranges between $15 \%$ and $35 \%$ [63]. These findings suggest that DDLT is not a feasible treatment modality for the majority of HCC patients. To overcome long waiting lists, disease progression and the dropout rate for LT, many centers tend to use "bridging" non-transplant therapies (e.g., liver resection, radiofrequency ablation, TACE) if the median waiting time for LT is more than 6 months [64-66]. These locoregional treatments may also play a critical role in successful down-staging in patients with HCC initially 


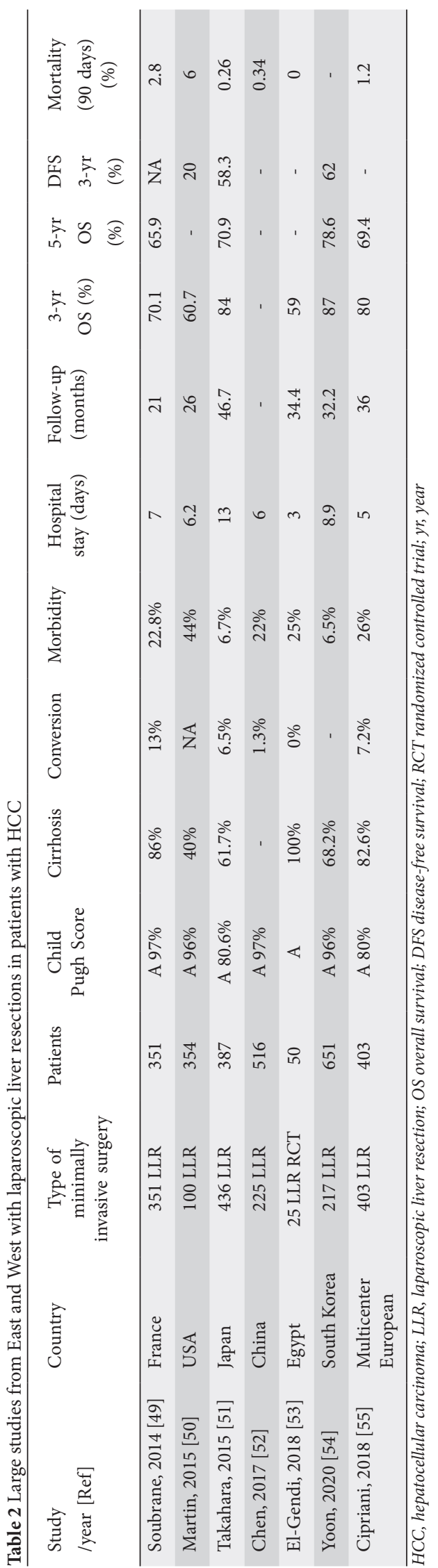

beyond the MC (i.e., macrovascular invasion) [64]. As the shortage of donors compared with the number of patients in need of transplantation is a serious and persistent problem worldwide, LDLT is emerging as an additional therapeutic option. Historically, the first report of successful LDLT was by Raia at the University of Sao Paulo in July 1989 [67]. LDLT as a treatment for HCC is especially popular in most Asian countries, given the difficulties in organ procurement from deceased donors [68]. LDLT is currently the only innovation that significantly expands the limited donor pool, as the growing demand for organs is not met by the available deceased liver grafts. HCC comprises over one third of the indications for LT in Asia, as compared with 10-20\% in Europe and the USA [69]. Even in Western countries, the necessity for LDLT is well established, in particular for more advanced HCC patients, who are disadvantaged by current allocation algorithms for grafts from deceased donors [70].

The application of LDLT has several advantages: 1) the transplantation can be performed on an elective basis before serious decompensation of the recipient or tumor growth. Waiting time can be very short in specialized centers, where LDLT is performed within a median of 44 days [71]. Therefore, LDLT for patients with HCC is a good option, minimizing the risk of dropout; 2) grafts are in excellent condition and complications due to preservation injury are absent; and 3) LDLT with relative donors has the potential to provide immunological benefits and thus reduce rejection episodes because of genetic similarities between the donor and the recipient. As a result, LDLT, which provides an excellent alternative for patients waiting for DDLT, has increased dramatically, especially in eastern countries [72].

However, LDLT presents with potential risks for posttransplant HCC recurrence that could impair recurrence-free survival, mainly for patients who do not fulfil the MC [73,74]. It is unclear whether this difference is due to a specific biological effect unique to the LDLT procedure, or to other factors such as patient selection. Firstly, the growth factors that mediate the regeneration of the liver graft after LDLT may potentiate HCC recurrence. The relatively small size of LDLT grafts may lead to additional mechanical injury at the start of the reperfusion process, and angiogenesis and cell division signaling pathways may be initiated more frequently. This rapid graft regeneration in LDLT has been implicated in a potential acceleration of tumor growth. Furthermore, an aggressive or rapidly progressive HCC biology may not be recognized during the short waiting time for transplantation. Because of the increased technical complexity of the LD allograft, LD recipients also have higher complication rates in comparison to diseased LT [75]. These postoperative complications following LDLT include bleeding, hepatic artery thrombosis, higher rates of biliary complications (biloma, cholangitis, etc.) and late biliary strictures. Moreover, another common factor limiting LDLT is represented by small-for-size syndrome (SFSS). SFSS can be defined [76] as functional liver impairment during the first postoperative period, as evidenced by coagulopathy, cholestasis, encephalopathy and ascites, and can lead to catastrophic consequences and increase mortality. 
Finally, the ethical dilemma of performing hepatectomy in a healthy donor with the potential for poor outcomes in the LDLT recipient is an existing drawback of this procedure (median rate for donor morbidity $16.1 \%$, mainly postoperative biliary complications, less than $0.6 \%$ mortality) [77]. Over the last decade, a few large multicenter studies and meta-analyses have provided a sufficient description of how methods and baseline characteristics are related to overall patient survival and recurrence rates in both LDLT and DDLT patients. Closely matched patients who underwent LDLT or DDLT for HCC have demonstrated equivalent outcomes, and it can be concluded that there were no disadvantages with LDLT compared to DDLT regarding overall and recurrence-free survival. In this review we quote some of the largest (more than 45 participants who received LDL graft) and latest comparative studies between LDLT and DDLT for patients with HCC, 4 from western centers and 6 from Asia, summarized in Table 3 [73,78-86]. Most of them report comparable results between LDLT and DDLT. However, the results of the A2ALL cohort study [73] found a higher rate of recurrence within 5 years in LDLT compared to DDLT (38\% vs. $11 \%, \mathrm{P}=0.0004)$, but there was a clear tendency toward more aggressive tumor characteristics in the LDLT group.

The waiting list for transplantation with a deceased donor's liver graft seems to jeopardize recipients with potentially curable HCCs, given the constant risk of cancer progression beyond accepted staging criteria. LDLT diminishes not only waiting list mortality but also dropout rates due to tumor progression beyond the established criteria for DDLT, which are usually very restrictive [87]. Moreover, improvements in patient selection and novel techniques for the endoscopic management of biliary complications post-LDLT (especially biliary anastomotic strictures) will ensure the success rate of LDLT in terms of survival. Furthermore, allograft selection, potential use of inflow modification, and optimization of outflow are all strategies that should be used to decrease the incidence of SFSS. Finally, studies from many Asian centers demonstrate that, with the incorporation of biological markers in the selection criteria to eliminate biologically aggressive HCCs, LDLT may have a significantly improved overall and disease-free survival for HCC patients [88].

In conclusion, LDLT could be a beneficial management strategy in HCC treatment. New RCTs are required to reevaluate preoperative screening, postoperative surveillance, and downstaging protocols in HCC patients receiving LDLT, to ensure optimal and timely therapy. Lastly, therapeutic options for patients with advanced HCC before LDLT need to be further expanded to improve survival.

\section{Molecular markers in HCC}

HCC is characterized by considerable phenotypic and molecular heterogeneity. During the last 2 decades, there has been an increasing understanding of the abundant molecular alterations in HCC; however, this has not been translated into

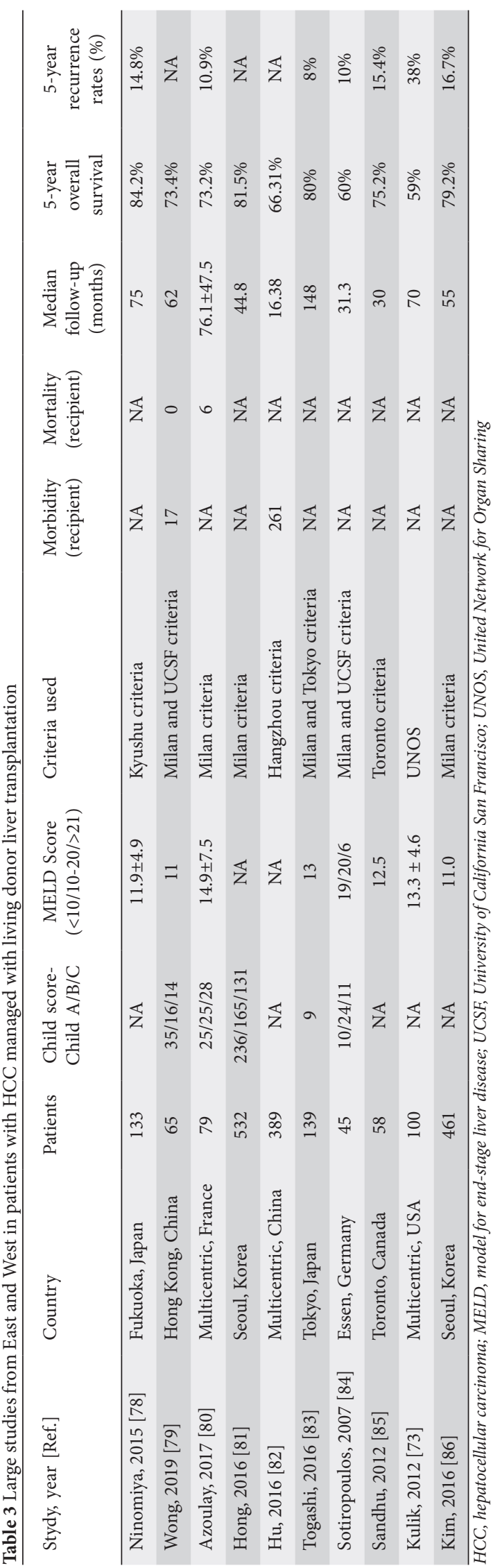


prognostic assessment or therapeutic decision making [10]. Knowledge of the cellular composition of tumors and the corresponding tumor microenvironment will enable the development of prognostic and predictive biomarkers that can be utilized in routine clinical practice [89]. Biomarkers represent an easy and noninvasive way to detect HCC at early stages and have the potential to estimate disease prognosis and recurrence. In spite of the numerous efforts to find molecules as possible biomarkers, there is not a single ideal marker in HCC.

Alpha-fetoprotein (AFP) is a serum glycoprotein first recognized as a marker for HCC more than 40 years ago and has since been used for early HCC detection. However, normal AFP levels are present in $30 \%$ of HCC patients at the time of diagnosis and usually remain low, even with advanced HCC [90]. AFP $>400 \mathrm{ng} / \mathrm{mL}$ is considered diagnostic for HCC, although less than half of the patients may have such high levels. With values of that magnitude, the specificity of AFP for HCC is close to $100 \%$, but the sensitivity falls below $45 \%[90,91]$. For this reason, it is imperative to find other, more sensitive molecular markers for the diagnosis and early identification of recurrence of HCC. Towards this direction new molecules have been investigated.

More specifically, autophagy molecules seem to have a key role in HCC. Autophagy is an evolutionarily conserved lysosome-dependent catabolic process that degrades cells components in order to recycle substrates to exert optimally and adapt to tough circumstances. It is a critical cellular homeostatic mechanism with stress resistance, immunity, antiaging, and anti-tumor effects [92]. Basal autophagy acts as a tumor suppressor by maintaining genomic stability in normal cells. However, once a tumor is established, unbalanced autophagy will contribute to carcinoma cell survival in the tumor microenvironment and in turn promote tumor growth and development.

Beclin-1, LC3-II and p62 are autophagy genes that can be used as prognostic molecules for HCC [93]. According to multicenter studies, increased autophagy has been detected in advanced liver cancer and is closely related to malignant transformation and a low survival rate in HCC patients. Moreover, autophagy contributes to the chemoresistance of HCC cells $[94,95]$. Fig. 2 depicts the role of autophagy in HCC and the implementations it may have in the diagnosis, prognosis and early detection of recurrence in HCC.

LC3-II, a widely used autophagic biomarker, was revealed to play a significant role in the development of cancer and is associated with the poor survival of cancer patients. Wu et al reported that LC3-II was overexpressed in the tumor region, compared with normal adjacent tissues, and the expression levels of LC3-II were positively related with vascular invasion and lymph node metastasis of HCC patients [96].

The expression level of Beclin 1 may be a valuable biomarker for HCC. A study of 103 primary HCC patients showed that the levels of Beclin 1 were significantly lower in HCC tissues than in adjacent tissues ( $72.8 \%$ vs. $89.5 \%)$. Beclin 1 may be a valuable prognostic marker of liver cancer, and loss or lower expression of Beclin 1 may suggest an inferior prognosis for HCC [97].

Moreover, p62 accumulation, induced by loss of autophagy, contributes to hepatic tumor formation $[93,98]$. Autophagy deficiency causes accumulation of p62, resulting in development of HCC.

Another serological and histochemical marker that is specific for HCC is GPC3. GPC3 is a member of the glypican family and belongs to a group of heparan sulfate proteoglycans bound to the outer surface of the cell membrane. GPC3 has been detected in the placenta and fetal liver, but not in other adult organs [99]. A dramatic elevation of GPC3 expression has been reported in a large proportion of HCCs, whereas its expression has been shown to be less frequent in preneoplastic or entirely absent in non-neoplastic liver tissue. Comparing the overall survival between GPC3-positive and GPC3-negative HCC patients, the GPC3-positive patients had a significantly lower 5-year survival rate than the GPC3-negative patients (54.5\% vs. 87.7\%) [100]. According to previous reports, higher levels of GPC3 expression were observed in moderately or poorly differentiated HCC, while at the same time GPC3 expression seems to be significantly linked to a poor prognosis after surgical resection in HCC patients [101].

Another biomarker that seems to be involved in the development and progression of HCC is $\beta$-catenin. The Wnt $/ \beta$ catenin pathway has shown significant promise as a potential target for novel molecular therapies. Moreover, $\beta$-catenin mutation seems to be related to the prognosis of HCC. Specifically, according to research studies, $\beta$-catenin protein expression is significantly greater in HCC tissue (72.94\%) compared with normal and cirrhotic liver $(22.35 \%$ and $26.67 \%$, respectively) [102,103]. B-catenin mutation is more frequently seen in earlier stages of HCC (I and II). Aberrant Wnt/ $\beta$ catenin signaling has been shown to be common in HCC tumors and has a clinical impact on tumor behavior, prognosis and response to treatment. As a result, $\mathrm{Wnt} / \beta$-catenin may be a promising target for future HCC therapies [104]. Currently, there are 2 clinical phase I/II trials studying the use of agents (such as PRI-724 and OMP-18R5) that specifically target the $\beta$-catenin signaling pathway to treat solid tumors and myeloid malignancies [105]. Recently, the Japanese Society of Hepatology's guidelines added to their recommendations the use of AFP in combination with des-carboxyprothrombin and AFP-L3 [106]. The guidelines of the American Association for the Study of Liver Diseases (AASLD) and the European Association for the Study of the Liver (EASL) include CD34, CK7, glypican 3, HSP-70, and glutamine synthetase staining to improve diagnostic accuracy [10,107]. Regarding prognosis, the EASL recommends the use of AFP levels, VEGF and angiopoietin 2 as independent prognostic biomarkers, in addition to the possible implementation of keratin 19 and EpCAM because of their correlation with worse outcomes in patients with HCC $[10,108]$. Lastly, cell free DNA (cfDNA) and circular RNAs (such as cSMARCA5 and circZKSCAN1) have been used in clinical trials as biomarkers for the diagnosis, early recurrence detection and treatment of HCC $[109,110]$. 


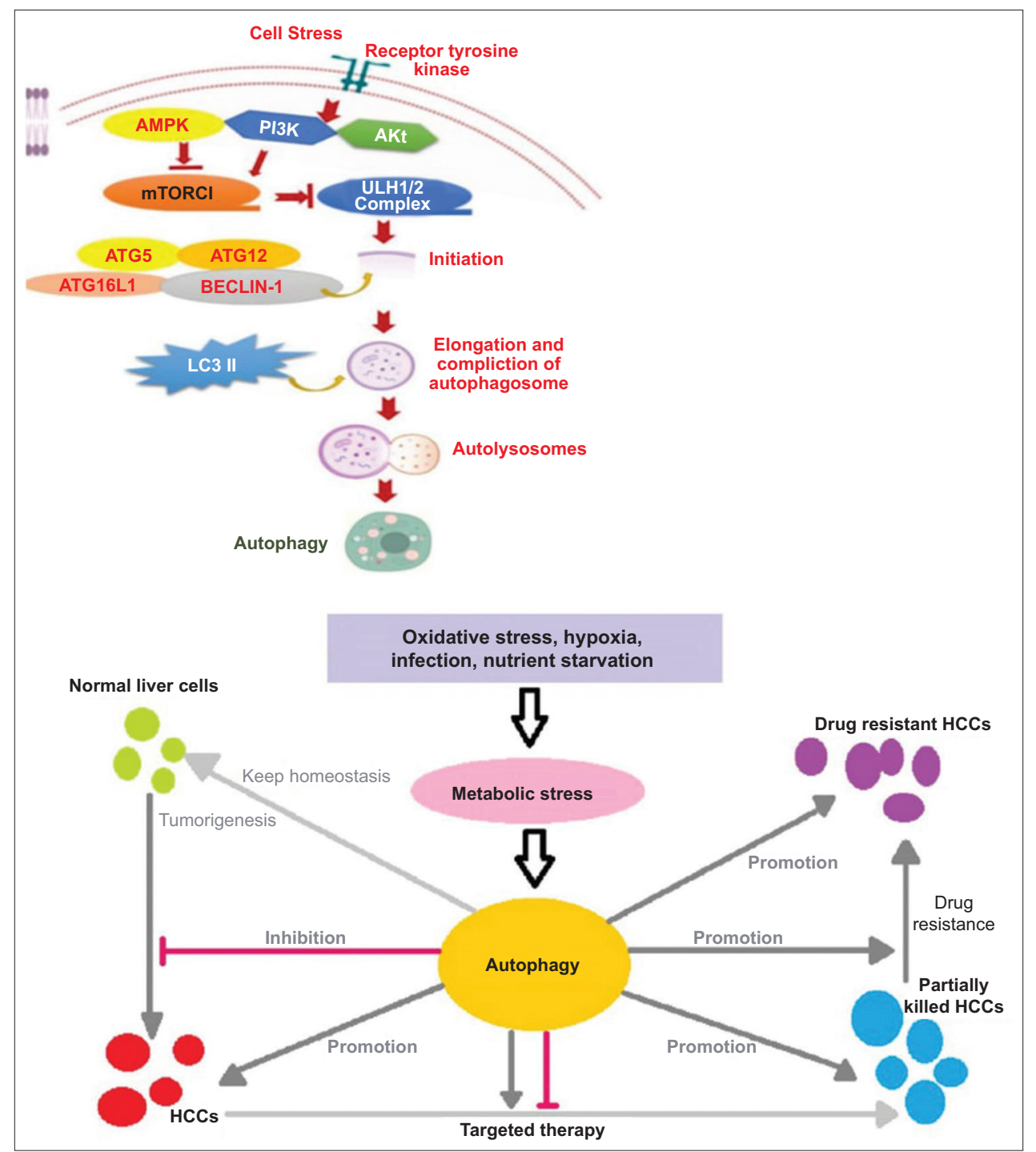

Figure 2 Autophagy reduction contributes to tumor initiation, and increased autophagy allows cancer cells to survive under stress conditions. The function of autophagy in liver cancer is a topic of concern, and it plays multiple roles in different situations. In normal liver cells, basal autophagy is involved in maintaining liver homeostasis. Once hepatocellular carcinoma (HCC) is established, autophagy plays a promotional role in tumor development, metastasis, and therapeutic resistance. Thus, appropriate autophagy inhibition could effectively suppress HCC growth and metastasis. However, in targeted HCC therapy, the role of autophagy is uncertain, for either inhibition or promotion, according to the different characteristics of agents. Autophagy induction at the tumor development stage promotes resistance to cancer therapy, while inhibition of autophagy promotes cancer cell death during cancer therapy

Most recent publications suggest that the combination of 2 or more biomarkers and additional demographic information can improve their sensitivity, specificity and predictive value. However, further research is needed to assess the use of biomarkers in clinical practice in patients with HCC. Table 4 summarizes the principal biomarkers of HCC with future perspectives.

Early diagnosis of HCC and detection of disease recurrence may offer patients the opportunity to undergo therapeutic interventions (such as hepatectomy or LT). For this reason, biomarkers are inextricably linked to the surgical management of patients with HCC. As mentioned above, the specificity of AFP for HCC is close to $100 \%$, but the sensitivity falls below $45 \%$, making it imperative to find other more sensitive molecular markers for the diagnosis and early identification of recurrence of HCC. Towards this direction, molecules such as GPC3, cfDNA and circular RNAs can be used as biomarkers for diagnosis, early recurrence detection and treatment of HCC. The above molecules can be isolated from both tissue and blood samples, whereas autophagy molecules and wnt $/ \beta$ catenin are both isolated from tissue. This feature does not contraindicate their use in clinical practice, since their levels are related with HCC prognosis and further future therapeutic decision. 
Table 4 Principal HCC biomarkers with future perspectives for early diagnosis, prognosis and disease recurrence

\begin{tabular}{|c|c|c|c|c|c|}
\hline & $\begin{array}{l}\text { Molecular } \\
\text { Marker }\end{array}$ & $\begin{array}{l}\text { Tissue or blood } \\
\text { isolation }\end{array}$ & Study / year, [Ref] & Design, Period & Patients \\
\hline \multirow{3}{*}{$\begin{array}{l}\text { Early } \\
\text { diagnosis }\end{array}$} & AFP & Blood & Mancebo, 2012 [91] & Retrospective study September 1992-March 2010 & 450 \\
\hline & cSMARCA5 & Tissue & Yu, 2018 [109] & Retrospective study 2010-2011, 2016-2017 & 208 \\
\hline & circZKSCAN1 & Tissue & Zhu, 2019 [110] & Retrospective study 2009-2010 & 112 \\
\hline \multirow[t]{7}{*}{ Prognosis } & LC3-II & Tissue & Wu, $2014[96]$ & Retrospective study 09/2003-09/2010 & 156 \\
\hline & Beclin-1 & Tissue & Qin, 2018 [97] & Meta-analysis 10 studies & 1086 \\
\hline & P62 & Tissue & Xiang, 2017 [98] & Retrospective study February 2013-February 2014 & 108 \\
\hline & AFP & Blood & Bai, 2017 [90] & Retrospective study 1988-2013 & 38,820 \\
\hline & GPC3 & Tissue & Kaseb, 2016 [101] & Retrospective study March 1996- September 2012 & 101 \\
\hline & $\beta$-catenin & Tissue & Rebouissou, 2016 [102] & Retrospective study 1997-2010 & 559 \\
\hline & keratin19 & Tissue & Lee, 2012 [108] & Retrospective study January 2000- August 2010 & 204 \\
\hline \multirow[t]{3}{*}{ Recurrence } & cSMARCA5 & Tissue & Yu, 2018 [109] & Retrospective study 2010-2011, 2016-2017 & 208 \\
\hline & circZKSCAN1 & Tissue & Zhu, $2019[110]$ & Retrospective study 2009-2010 & 112 \\
\hline & $\beta$-catenin & Tissue & Rebouissou, 2016 [102] & Retrospective study $1997-2010$ & 559 \\
\hline
\end{tabular}

HCC, hepatocellular carcinoma

\section{Concluding remarks}

The management of HCC is complex and requires a multidisciplinary, but at the same time individualized approach. There have been very significant advances over the last 15 years in the surgical management of HCC, such as aggressive surgical management of selected patients with intermediate and advanced stage, application of minimally invasive surgery and LDLT. In the future, the further development of interventional radiology, which will increase resectability, along with the judicious use of molecular markers and the expanded application of minimally invasive surgery and LDLT, will increase the options in many patients with HCC, who will achieve long survival with a good quality of life.

\section{References}

1. Bray F, Ferlay J, Soerjomataram I, Siegel RL, Torre LA, Jemal A. Global cancer statistics 2018: GLOBOCAN estimates of incidence and mortality worldwide for 36 cancers in 185 countries. CA Cancer J Clin 2018;68:394-424.

2. Haque E, Karim M-R, Teeli AS, et al. Molecular mechanisms underlying hepatocellular carcinoma induction by Aberrant NRF2 activation-mediated transcription networks: interaction of NRF2-KEAP1 controls the fate of hepatocarcinogenesis. Int $\mathrm{J} \mathrm{Mol}$ Sci 2020;21:5378.

3. Anastasopoulos NT, Lianos GD, Tatsi V, Karampa A, Goussia A, Glantzounis GK. Clinical heterogeneity in patients with nonalcoholic fatty liver disease-associated hepatocellular carcinoma. Expert Rev Gastroenterol Hepatol 2020;14:1025-1033.

4. Mungroop TH, Geerts BF, Veelo DP, et al. Fluid and pain management in liver surgery (MILESTONE): A worldwide study among surgeons and anesthesiologists. Surgery 2019;165:337-344.

5. Gasteiger L, Eschertzhuber S, Tiefenthaler W. Perioperative management of liver surgery-review on pathophysiology of liver disease and liver failure. Eur Surg 2018;50:81-86.

6. Tzimas P, Prout J, Papadopoulos G, Mallett SV. Epidural anaesthesia and analgesia for liver resection. Anaesthesia 2013;68:628-635.

7. Agarwal V, Divatia JV. Enhanced recovery after surgery in liver resection: current concepts and controversies. Korean $J$ Anesthesiol 2019;72:119-129.

8. Chen LT, Martinelli E, Cheng AL, et al. Pan-Asian adapted ESMO Clinical Practice Guidelines for the management of patients with intermediate and advanced/relapsed hepatocellular carcinoma: a TOS-ESMO initiative endorsed by CSCO, ISMPO, JSMO, KSMO, MOS and SSO. Ann Oncol 2020;31:334-351.

9. Llovet JM, Bruix J. Systematic review of randomized trials for unresectable hepatocellular carcinoma: Chemoembolization improves survival. Hepatology 2003;37:429-442.

10. European Association for the Study of the Liver. EASL Clinical Practice Guidelines: Management of hepatocellular carcinoma. $J$ Hepatol 2018;69:182-236.

11. Lo CM, Ngan H, Tso WK, et al. Randomized controlled trial of transarterial lipiodol chemoembolization for unresectable hepatocellular carcinoma. Hepatology 2002;35:1164-1171.

12. Llovet JM, Real MI, Montaña X, et al; Barcelona Liver Cancer Group. Arterial embolisation or chemoembolisation versus symptomatic treatment in patients with unresectable hepatocellular carcinoma: a randomised controlled trial. Lancet 2002;359:1734-1739.

13. Malagari K, Moschouris H, Kiakidis T, et al. Five-years outcome analysis of 142 consecutive hepatocellular carcinoma patients treated with doxorubicin eluting microspheres $30-60 \mathrm{~lm}$ : results from a single-centre prospective phase II Trial. Cardiovasc Intervent Radiol 2019;42:1551-1562.

14. Torzilli G, Belghiti J, Kokudo N, et al. A snapshot of the effective indications and results of surgery for hepatocellular carcinoma in tertiary referral centers: is it adherent to the EASL/AASLD recommendations?: an observational study of the HCC EastWest study group. Ann Surg 2013;257:929-937.

15. Glantzounis GK, Paliouras A, Stylianidi MC, et al. The role of liver resection in the management of intermediate and advanced 
stage hepatocellular carcinoma. A systematic review. Eur J Surg Oncol 2018;44:195-208.

16. Bruix J, Qin S, Merle P, et al; RESORCE Investigators. Regorafenib for patients with hepatocellular carcinoma who progressed on sorafenib treatment (RESORCE): a randomised, double-blind, placebo-controlled, phase 3 trial. Lancet 2017;389:56-66.

17. Ikai I, Kudo M, Arii S, et al. Report of the 18th follow-up survey of primary liver cancer in Japan. Hepatol Res 2010;40:1043-1059.

18. Shi J, Lai EC, Li N, et al. A new classification for hepatocellular carcinoma with portal vein tumor thrombus. J Hepatobiliary Pancreat Sci 2011;18:74-80.

19. Cerrito L, Annicchiarico BE, Iezzi R, Gasbarrini A, Pompili M, Ponziani FR. Treatment of hepatocellular carcinoma in patients with portal vein tumor thrombosis: Beyond the known frontiers. World J Gastroenterol 2019;25:4360-4382.

20. Takemura N, Aoki T, Hasegawa K, et al. Hepatectomy for hepatocellular carcinoma after perioperative management of portal hypertension. Br J Surg 2019;106:1066-1074.

21. Yopp AC, Mansour JC, Beg MS, et al. Establishment of a multidisciplinary hepatocellular carcinoma clinic is associated with improved clinical outcome. Ann Surg Oncol 2014;21:1287-1295.

22. LubelJS, Roberts SK, StrasserSI, et al. Australian recommendations for the management of hepatocellular carcinoma: a consensus statement. Med J Aust 2020 Dec 13 [Online ahead of print]. doi: 10.5694/mja2.50885

23. Reich H, McGlynn F, DeCaprio J, Budin R. Laparoscopic excision of benign liver lesions. Obstet Gynecol 1991;78:956-958.

24. Buell JF, Cherqui D, Geller DA, et al; World Consensus Conference on Laparoscopic Surgery. The international position on laparoscopic liver surgery: the Louisville Statement, 2008. Ann Surg 2009;250:825-830.

25. Wakabayashi G, Cherqui D, Geller DA, et al. Recommendations for laparoscopic liver resection: a report from the second international consensus conference held in Morioka. Ann Surg 2015;261:619-629.

26. Han HS, Cho JY, Kaneko H, et al. Expert panel statement on laparoscopic living donor hepatectomy. Dig Surg 2018; 35:284-288.

27. Cho JY, Han HS, Wakabayashi G, et al. Practical guidelines for performing laparoscopic liver resection based on the second international laparoscopic liver consensus conference. Surg Oncol 2018;27:A5-A9.

28. Ciria R, Gomez-Luque I, Ocaña S, et al. A systematic review and meta-analysis comparing the short- and long-term outcomes for laparoscopic and open liver resections for hepatocellular carcinoma: updated results from the European guidelines meeting on laparoscopic liver surgery, Southampton, UK, 2017. Ann Surg Oncol 2019;26:252-263.

29. Di Sandro S, Danieli M, Ferla F, et al. The current role of laparoscopic resection for HCC: a systematic review of past ten years. Transl Gastroenterol Hepatol 2018;3:368.

30. Sotiropoulos G, Prodromidou A, Kostakis I, Machairas N. Metaanalysis of laparoscopic vs open liver resection for hepatocellular carcinoma. Updates Surg 2017;69:291-311.

31. Shehta A, Han HS, Yoon YS, Cho JY, Choi Y. Laparoscopic liver resection for hepatocellular carcinoma in cirrhotic patients: 10year single-center experience. Surg Endosc 2016;30:638-648.

32. Wu X, Huang Z, Lau WY, et al. Perioperative and long-term outcomes of laparoscopic versus open liver resection for hepatocellular carcinoma with well-preserved liver function and cirrhotic background: a propensity score matching study. Surg Endosc 2019;33:206-215.

33. Zheng B, Zhao R, Li X, Li B. Comparison of laparoscopic liver resection for lesions located in anterolateral and posterosuperior segments: a meta-analysis. Surg Endosc 2017;31:4641-4648.
34. Kwon Y, Han HS, Yoon YS, Cho JY. Are large hepatocellular carcinomas still a contraindication for laparoscopic liver resection? J Laparoendosc Adv Surg Tech A 2015;25:98-102.

35. Fu XT, Tang Z, Shi YH, et al. Laparoscopic versus open left lateral segmentectomy for large hepatocellular carcinoma: a propensity score-matched analysis. Surg Laparosc Endosc Percutan Tech 2019;29:513-519.

36. Ibuki S, Hibi T, Tanabe M, Geller DA, Cherqui D, Wakabayashi G; INSTALL-2 Collaborative Study Group. Short-term outcomes of "difficult" laparoscopic liver resection at specialized centers: report from INSTALL (International Survey on Technical Aspects of Laparoscopic Liver Resection)-2 on 4478 patients. Ann Surg 2020 Sep 1 [Online ahead of print]. doi: 10.1097/ SLA.0000000000004434

37. Santambrogio R, Barabino M, De Nicola E, Galfrascoli E, Giovenzana M, Zappa MA. Laparoscopic ablation therapies for hepatocellular carcinoma: could specific indications for the laparoscopic approach influence the effectiveness? Updates Surg 2020;72:435-443.

38. Machairas N, Papaconstantinou D, Stamopoulos P, et al. The emerging role of laparoscopic liver resection in the treatment of recurrent hepatocellular carcinoma: a systematic review. Anticancer Res 2018;38:3181-3186.

39. Wakabayashi T, Felli E, Memeo R, et al. Short-term outcomes of laparoscopic repeat liver resection after open liver resection: a systematic review. Surg Endosc 2019;33:2083-2092.

40. Urade T, Sawa H, Iwatani Y, et al. Laparoscopic anatomical liver resection using indocyanine green fluorescence imaging. Asian $J$ Surg 2020;43:362-368.

41. Giulianotti PC, Sbrana F, Bianco FM, Addeo P. Robotassisted laparoscopic extended right hepatectomy with biliary reconstruction. J Laparoendosc Adv Surg Tech A 2010;20:159-163.

42. Efanov M, Alikhanov R, Tsvirkun V, et al. Comparative analysis of learning curve in complex robot-assisted and laparoscopic liver resection. HPB (Oxford) 2017;19:818-824.

43. Nota CL, Rinkes IHB, Hagendoorn J. Setting up a robotic hepatectomy program: a Western-European experience and perspective. Hepatobiliary Surg Nutr 2017;6:239-245.

44. Hu L, Yao L, Li X, Jin P, Yang K, Guo T. Effectiveness and safety of robotic-assisted versus laparoscopic hepatectomy for liver neoplasms: A meta-analysis of retrospective studies. Asian J Surg 2018;41:401-416.

45. Wang ZZ, Tang WB, Hu MG, et al. Robotic vs laparoscopic hemihepatectomy: a comparative study from a single center. $J$ Surg Oncol 2019;120:646-653.

46. Kingham TP, Leung U, Kuk D, et al. Robotic liver resection: a case-matched comparison. World J Surg 2016;40:1422-1428.

47. Caruso S, Patriti A, Ceccarelli G, Corrati A. Minimally invasive liver resection: has the time come to consider robotics a valid assistance? Hepatobiliary Surg Nutr 2018;7:195-198.

48. Magistri P, Tarantino G, Guidetti C, et al. Laparoscopic versus robotic surgery for hepatocellular carcinoma: the first 46 consecutive cases. J Surg Res 2017;217:92-99.

49. Soubrane O, Goumard C, Laurent A, et al. Laparoscopic resection of hepatocellular carcinoma: a French survey in 351 patients. HPB (Oxford) 2014;16:357-365.

50. Martin RC 2nd, Mbah NA, St Hill R, et al. Laparoscopic versus open hepatic resection for hepatocellular carcinoma: improvement in outcomes and similar cost. World J Surg 2015;39:1519-1526.

51. Takahara T, Wakabayashi G, Beppu T, et al. Long-term and perioperative outcomes of laparoscopic versus open liver resection for hepatocellular carcinoma with propensity score matching: a multi-institutional Japanese study. J Hepatobiliary Pancreat Sci 2015;22:721-727. 
52. Chen J, Li H, Liu F, Li B, Wei Y. Surgical outcomes of laparoscopic versus open liver resection for hepatocellular carcinoma for various resection extent. Medicine (Baltimore) 2017;96:e6460.

53. El-Gendi A, El-Shafei M, El-Gendi S, Shawky A. Laparoscopic versus open hepatic resection for solitary hepatocellular carcinoma less than $5 \mathrm{~cm}$ in cirrhotic patients: a randomized controlled study. J Laparoendosc Adv Surg Tech A 2018;28:302-310.

54. Yoon YI, Kim $\mathrm{KH}$, Cho $\mathrm{HD}$, et al. Long-term perioperative outcomes of pure laparoscopic liver resection versus open liver resection for hepatocellular carcinoma: a retrospective study. Surg Endosc 2020;34:796-805.

55. Cipriani F, Fantini C, Ratti F, et al. Laparoscopic liver resections for hepatocellular carcinoma. Can we extend the surgical indication in cirrhotic patients? Surg Endosc 2018;32:617-626.

56. Ferlay J, Soerjomataram I, Dikshit R, et al. Cancer incidence and mortality worldwide: sources, methods and major patterns in GLOBOCAN 2012. Int J Cancer 2015;136:E359-E386.

57. Forner A, Llovet JM, Bruix J. Hepatocellular carcinoma. Lancet 2012;379:1245-1255.

58. Lee KW, Yi NJ, Suh KS. Section 5. Further expanding the criteria for HCC in living donor liver transplantation: when not to transplant: SNUH experience. Transplantation 2014;97 Suppl 8:S20-S23.

59. Mazzaferro V, Regalia E, Doci R, et al. Liver transplantation for the treatment of small hepatocellular carcinomas in patients with cirrhosis. N Engl J Med 1996;334:693-699.

60. Mazzaferro V, Bhoori S, Sposito C, et al. Milan criteria in liver transplantation for hepatocellular carcinoma: an evidence-based analysis of 15 years of experience. Liver Transpl 2011;17 Suppl 2:S44-S57.

61. National Cancer Center (NCC), Goyang, Korea. 2018 Korean Liver Cancer Association-National Cancer Center Korea Practice Guidelines for the Management of Hepatocellular Carcinoma. Korean J Radiol 2019;20:1042-1113.

62. Yao FY, Bass NM, Nikolai B, et al. Liver transplantation for hepatocellular carcinoma: analysis of survival according to the intention-to-treat principle and dropout from the waiting list. Liver Transpl 2002;8:873-883.

63. Menahem B, Lubrano J, Duvoux C, et al. Liver transplantation versus liver resection for hepatocellular carcinoma in intention to treat: an attempt to perform an ideal meta-analysis. Liver Transpl 2017;23:836-844.

64. Assalino M, Terraz S, Grat M, et al. Liver transplantation for hepatocellular carcinoma after successful treatment of macrovascular invasion - a multi-center retrospective cohort study. Transpl Int 2020;33:567-575.

65. Na GH, Kim EY, Hong TH, You YK, Kim DG. Effects of loco regional treatments before living donor liver transplantation on overall survival and recurrence-free survival in South Korean patients with hepatocellular carcinoma. HPB (Oxford) 2016;18:98-106.

66. Llovet JM, Pavel M, Rimola J, et al. Pilot study of living donor liver transplantation for patients with hepatocellular carcinoma exceeding Milan Criteria (Barcelona Clinic Liver Cancer extended criteria). Liver Transpl 2018;24:369-379.

67. Raia S, Nery JR, Mies S. Liver transplantation from live donors. Lancet 1989;2:497.

68. Rela M, Reddy MS. Living donor liver transplant (LDLT) is the way forward in Asia. Hepatol Int 2017;11:148-151.

69. de Villa V, Lo CM. Liver transplantation for hepatocellular carcinoma in Asia. Oncologist 2007;12:1321-1331.

70. Han DH, Joo DJ, Kim MS, et al. Living donor liver transplantation for advanced hepatocellular carcinoma with portal vein tumor thrombosis after concurrent chemoradiation therapy. Yonsei Med J 2016;57:1276-1281.

71. Zhang HM, Shi YX, Sun LY, Zhu ZJ. Hepatocellular carcinoma recurrence in living and deceased donor liver transplantation: a systematic review and meta-analysis. Chin Med J (Engl) 2019;132:1599-1609.

72. Liang $\mathrm{W}$, Wu L, Ling $\mathrm{X}$, et al. Living donor liver transplantation versus deceased donor liver transplantation for hepatocellular carcinoma: a meta-analysis. Liver Transpl 2012;18:1226-1236.

73. Kulik LM, Fisher RA, Rodrigo DR, et al. Outcomes of living and deceased donor liver transplant recipients with hepatocellular carcinoma: results of the A2ALL cohort. Am J Transplant 2012;12:2997-3007.

74. Park MS, Lee KW, Suh SW, et al. Living-donor liver transplantation associated with higher incidence of hepatocellular carcinoma recurrence than deceased-donor liver transplantation. Transplantation 2014;97:71-77.

75. Park J, Choi GS, Gwak MS, et al. A retrospective analysis of reexploration after living donor right lobe liver transplantation: incidence, causes, outcomes, and risk factors. Transpl Int 2019;32:141-152.

76. Miller CM, Quintini C, Dhawan A, et al. The International Liver Transplantation Society Living Donor Liver Transplant Recipient Guideline. Transplantation 2017;101:938-944.

77. Benzing C, Schmelzle M, Oellinger R, et al. Living-donor liver transplant: an analysis of postoperative outcome and healthrelated quality of life in liver donors. Exp Clin Transplant 2018; 16:568-574.

78. Ninomiya M, Shirabe K, Facciuto ME, et al. Comparative study of living and deceased donor liver transplantation as a treatment for hepatocellular carcinoma. J Am Coll Surg 2015;220:297-304.

79. Wong TCL, Ng KKC, Fung JYY, et al. Long-term survival outcome between living donor and deceased donor liver transplant for hepatocellular carcinoma: intention-to-treat and propensity score matching analyses. Ann Surg Oncol 2019;26:1454-1462.

80. Azoulay D, Audureau E, Bhangui P, et al. Living or brain-dead donor liver transplantation for hepatocellular carcinoma: a multicenter, western, intent-to-treat cohort study. Ann Surg 2017;266:1035-1044.

81. Hong SK, Lee KW, Kim HS, Yoon KC, Yi NJ, Suh KS. Living donor liver transplantation for hepatocellular carcinoma in Seoul. Hepatobiliary Surg Nutr 2016;5:453-460.

82. Hu Z, Qian Z, Wu J, et al. Clinical outcomes and risk factors of hepatocellular carcinoma treated by liver transplantation: A multi-centre comparison of living donor and deceased donor transplantation. Clin Res Hepatol Gastroenterol 2016;40:315-326.

83. Togashi J, Akamastu N, Kokudo N. Living donor liver transplantation for hepatocellular carcinoma at the University of Tokyo Hospital. Hepatobiliary Surg Nutr 2016;5:399-407.

84. Sotiropoulos GC, Lang H, Nadalin S, et al. Liver transplantation for hepatocellular carcinoma: University Hospital Essen experience and metaanalysis of prognostic factors. J Am Coll Surg 2007;205:661-675.

85. Sandhu L, Sandroussi C, Guba M, et al. Living donor liver transplantation versus deceased donor liver transplantation for hepatocellular carcinoma: comparable survival and recurrence. Liver Transpl 2012;18:315-322.

86. Kim SH, Moon DB, Kim WJ, et al. Preoperative prognostic values of $\alpha$-fetoprotein (AFP) and protein induced by vitamin $\mathrm{K}$ absence or antagonist-II (PIVKA-II) in patients with hepatocellular carcinoma for living donor liver transplantation. Hepatobiliary Surg Nutr 2016;5:461-469.

87. Pavel MC, Sanchez Cabus S, Crespo G, et al. Role of adult living donor liver transplantation in the treatment of hepatocellular carcinoma within and beyond Milan criteria. Transplant Proc 2018;50:1386-1395.

88. Ogawa K, Takada Y. Living vs. deceased-donor liver transplantation for patients with hepatocellular carcinoma. Transl Gastroenterol Hepatol 2016;1:35.

89. Villanueva A, Minguez B, Forner A, Reig M, Llovet J. 
Hepatocellular carcinoma: novel molecular approaches for diagnosis, prognosis, and therapy. Annu Rev Med 2010;61:317328.

90. Bai DS, Zhang C, Chen P, Jin SJ, Jiang GQ. The prognostic correlation of AFP level at diagnosis with pathological grade, progression, and survival of patients with hepatocellular carcinoma. Sci Rep 2017;7:12870.

91. Mancebo A, González-Diéguez ML, Cadahía V, et al. Annual incidence of hepatocellular carcinoma among patients with alcoholic cirrhosis and identification of risk groups. Clin Gastroenterol Hepatol 2013;11:95-101.

92. Liu L, Liao JZ, HeXX, Li PY. The role of autophagy in hepatocellular carcinoma: friend or foe. Oncotarget 2017;8:57707-57722.

93. Kessler SM, Laggai S, Barghash A, et al. IMP2/p62 induces genomic instability and an aggressive hepatocellular carcinoma phenotype. Cell Death Dis 2015;6:e1894.

94. Vanzo R, Bartkova J, Merchut-Maya JM, et al. Autophagy role(s) in response to oncogenes and DNA replication stress. Cell Death Differ 2020;27:1134-1153.

95. Huang F, Wang BR, Wang YG. Role of autophagy in tumorigenesis, metastasis, targeted therapy and drug resistance of hepatocellular carcinoma. World J Gastroenterol 2018;24:4643-4651.

96. Wu DH, Jia CC, Chen J, et al. Autophagic LC3B overexpression correlates with malignant progression and predicts a poor prognosis in hepatocellular carcinoma. Tumour Biol 2014;35:12225-12233.

97. Qin Z, Yu X, Lin M, Wu J, Ma S, Wang N. Prognostic and clinicopathological value of Beclin-1 expression in hepatocellular carcinoma: a meta-analysis. World J Surg Oncol 2018;16:170.

98. Xiang X, Qin HG, You XM, et al. Expression of P62 in hepatocellular carcinoma involving hepatitis B virus infection and aflatoxin B1 exposure. Cancer Med 2017;6:2357-2369.

99. Shimizu Y, Mizuno S, Fujinami N, et al. Plasma and tumoral glypican-3 levels are correlated in patients with hepatitis $\mathrm{C}$ virusrelated hepatocellular carcinoma. Cancer Sci 2020;111:334-342.
100. Zhang J, Zhang M, Ma H, et al. Overexpression of glypican-3 is a predictor of poor prognosis in hepatocellular carcinoma an updated meta-analysis. Medicine (Baltimore) 2018;97:e11130.

101. Kaseb AO, Hassan M, Lacin S, et al. Evaluating clinical and prognostic implications of Glypican-3 in hepatocellular carcinoma. Oncotarget 2016;7:69916-69926.

102. Rebouissou S, Franconi A, Calderaro J, et al. Genotype-phenotype correlation of CTNNB1 mutations reveals different $B$-catenin activity associated with liver tumor progression. Hepatology 2016;64:2047-2061.

103. Khalaf AM, Fuentes D, Morshid AI, et al. Role of Wnt $/ \beta$-catenin signaling in hepatocellular carcinoma, pathogenesis, and clinical significance. J Hepatocell Carcinoma 2018;5:61-73.

104. Kim E, Lisby A, Ma C, et al. Promotion of growth factor signaling as a critical function of $\beta$-catenin during HCC progression. Nat Commun 2019;10:1909.

105. Jung YS, Park JI. Wnt signaling in cancer: therapeutic targeting of Wnt signaling beyond $\beta$-catenin and the destruction complex. Exp Mol Med 2020;52:183-191.

106. Kokudo N, Takemura N, Hasegawa K, et al. Clinical practice guidelines for hepatocellular carcinoma: The Japan Society of Hepatology 2017 (4th JSH-HCC guidelines) 2019 update. Hepatol Res 2019;49:1109-1113.

107. Heimbach JK, Kulik LM, Finn RS, et al. AASLD guidelines for the treatment of hepatocellular carcinoma. Hepatology 2018;67:358-380.

108. Lee J, Lee JW, Kim JM, Kim JK, Chung HJ, Kim YS. Prognosis of hepatocellular carcinoma expressing cytokeratin 19: Comparison with other liver cancers. World J Gastroenterol 2012;18:4751-4757.

109. Yu J, Xu QG, Wang ZG, et al. Circular RNA cSMARCA5 inhibits growth and metastasis in hepatocellular carcinoma. $J$ Hepatol 2018;68:1214-1227.

110. Zhu YJ, Zheng B, Luo GJ, et al. Circular RNAs negatively regulate cancer stem cells by physically binding FMRP against CCAR1 complex in hepatocellular carcinoma. Theranostics 2019;12:3526-3540. 